\title{
The use of virtual reality three-dimensional simulation technology in nursery school teacher training for the understanding of children's cognitive perceptions
}

\author{
Yaacov J. Katz \\ School of Education, Bar-Ilan University, Ramat-Gan 52900, Israel; katzya@mail.biu.ac.il
}

\begin{abstract}
This study examined the effectiveness and efficiency of a three-dimensional virtual reality simulation model designed to train nursery school teachers in the understanding of nursery school children's cognitive perceptions. An experimental group of 45 nursery school teachers underwent 20 hours of virtual reality simulation as opposed to 20 hours of workshop activity experienced by 44 nursery school teachers in a comparable control group. Both methodologies were designed to promote improved understanding of children's cognitive perceptions. After the training sessions the teachers were observed in their nursery school work over a period of two days by three nursery school supervisors who evaluated the research subjects' understanding of nursery school teacher's cognitive perceptions.
\end{abstract}

Statistical analysis of the data indicate that the nursery school teachers who were trained through the virtual reality simulation technology were significantly more understanding of children's cognitive perceptions and needs than those trained through the workshop method. Thus, in light of the results of the study, it is suggested that teacher trainers should favourably consider using virtual reality simulation models in the training of nursery school teachers in order to maximise the effectiveness of the teacher training process.

Key words: learning styles, teacher education

\section{INTRODUCTION}

Information and Communication Technology (ICT) has the potential to significantly contribute to the improvement of learning and teaching at all

The original version of this chapter was revised: The copyright line was incorrect. This has been corrected. The Erratum to this chapter is available at DOI: 10.1007/978-0-387-35668-6_17 
levels in the educational system. The development of highly sophisticated computer-based tools provides the educational system with rich potential for the development of more effective and efficient learning and teaching which, if successfully utilised, could lead to the hoped for educational revolution.

\section{VIRTUAL REALITY}

Among the state-of-the-art technological packages that have been made available to educators and learners are virtual reality (VR) technologies. Turman and Matton (1994) defined virtual reality as a highly interactive computer-based multimedia environment in which the user becomes a fullfledged partner in a virtual world. When experiencing virtual reality the user becomes an immersed participant in the computer-generated VR program. Bricken and Byrne (1992) stated that in order to enter into the world of virtual reality the learner wears a computerised helmet and gloves, which allow him/her to see, hear, and touch other objects in the virtual world. When the learner enters the virtual world s/he usually perceives the virtual world as a place in which s/he is physically immersed (Henry 1992). Regain and Shebliske (1992) reported that learners who enter a virtual world undergo physical and emotional experiences very similar to those that exist in the physical world.

\subsection{Simulation}

One of the major methodologies used in virtual reality is that of simulation and modelling (Van Weert 1995). Educational computer simulations are based on dynamic interactions between the learner and a computer program, and may be defined as that part of the modelling process which involves the execution of a model by the learner. The learner experiments with the simulated phenomenon by observing and analysing interactions between him/herself and the modelled phenomenon (Baranauskas and De Oliveira 1995). A number of studies have indicated that simulation is a rich and sophisticated computer-based learning environment that allows the learner to experience the learning situation in an encompassing, motivating and novel world. Simulation systems mimic the phenomenon under study and the learner's typical role is to discover, through a process of investigation, the rules that govern the phenomenon. The learner enters a powerful learning environment and engages in a cycle of expression, evaluation, and reflection (Schecker 1993). White (1993) described how learners who undertook animated simulation tasks in the physics of velocity were able to overcome common misconceptions that 
existed after studying in a traditional non-simulation environment, and Leary (1995) reported that a number of studies have indicated that learners' educational achievements through the teaching medium of simulation are higher than achievements attained after undergoing other non-simulation methods of instruction.

Cumming, Zangari and Thomason (1995) postulated that there are a number of basic assumptions that underlie the presumption that the learner will be able to derive more benefit from computer-based simulation than from other learning environments. These assumptions conclude that:

- the learner is capable of taking initiative and exploring - the simulated phenomenon provides a rich platform for the activities;

- learner engagement is all important - in simulation learner engagement is maximised by a sophisticated three-dimensional interface design that provides the learner with motivating as well as challenging activities;

- learning may be regarded as conceptual change - simulation facilitates conceptual change more efficiently than many other learning environments; learning is superior when the learner can experience both abstract and concrete aspects of the content under study - in simulations both abstract and concrete learning take place.

\section{TEACHERS' UNDERSTANDING OF CHILDREN'S COGNITIVE PERCEPTIONS}

Deen (1995) indicated that teaching in general is a process of understanding the pupils' cognitive perceptions. Teachers who are able to acquire this understanding are those who best cognitively empathise with their pupils, and are best equipped to assist them in the development of learning and problem-solving behaviour. According to data based on a longitudinal investigation conducted by Kikas (1998), teachers who understand their pupils' cognitive perceptions are more capable of assisting the pupils in problem-solving behaviour than teachers who have a lower level of understanding. Kikas confirmed that effective teaching is based on the confidence of teachers that they are able to understand the cognitive perceptions of their pupils and are consequently able to efficiently promote positive cognitive and problem-solving behaviours among their pupils. Calvert and Henderson (1994) stated that in order to equip teachers more comprehensively for their teaching tasks, they should undergo training in understanding the cognitive perceptions of their pupils. Bell (1998) concurred and added that effective teachers offer their pupils a varied range of rich cognitive experiences based on their deep understanding of their pupils' cognitive perceptions. Thus, in light of the evidence, it is logical to 
propose that teacher-training methods that are able to significantly contribute to teachers' familiarity with pupils' cognitive perceptions are the most effective and efficient.

\subsection{Teachers' understanding of nursery school children's cognitive perceptions}

Katznelson (1993) emphasised the potential traumas associated with the transition of the child from home or baby care centre to the more formal educational framework of the nursery school. The change that is experienced by the young child during the course of the transition can cause anxiety, fear, regression and even disorientation. Katznelson also indicated that the quality of assistance the child receives from the nursery school teacher in order to alleviate the problems associated with the transition to the new formal educational framework is dependent on the nursery school teacher's ability to fully understand the child's cognitive perceptions and to empathise with him/her.

Davies (1991) also addressed the problems that children entering nursery school may experience when entering their new educational framework, and pointed out that the nursery school is the first educational framework that makes basic cognitive demands of children and initially they may have difficulty coping with those demands. He also said that the better equipped a nursery school teacher is to deal with the cognitive perceptions of children beginning their nursery school year, the more competent the teacher is in contributing to the alleviation of children's problems. Rubinstein (1990) postulated that an important aspect of nursery school teachers' educational roles is to familiarise children with the cognitive demands of the new nursery school environment in order to allay anxieties and fear induced by the transition into a new and potentially threatening complex learning situation.

In his theory of cognitive development Piaget (1978) described in detail the different developmental stages that a child goes through from birth until late adolescence, during which the child's cognitive ability steadily develops. Basing their work on Piaget's developmental model, Glover and Bruning (1987) described the cognitive development and perceptions of the nursery school child. They indicated that nursery school children do not perceive their environment objectively and their perceptions are different from those of adults. Nursery school children categorise objects while using unitary characteristics, and are unable to view the cognitive world from a viewpoint other than their own because their reasoning tends to be egocentric. Adult cognition is based on formal operations, abstract thought and multifaceted categorisation of situations. Thus adult reasoning does not 
remotely resemble the cognitive patterns of a child and will not be effective when trying to understand the nursery school child's cognitive perceptions because of the vast developmental gap that exists between the two age groups. Phillips (1975) remarked that adults wishing to understand young children's cognitive perceptions must set aside their cognitive models and attempt to enter children's cognitive world. Without competent adult understanding of the nursery school child's cognitive processes, the cognitive gap between young children and adults will strongly mitigate against mutual understanding and perceptions.

\section{AIM OF THE RESEARCH PROJECT}

From the review of the literature it is apparent that teachers who better understand pupils' cognitive perceptions are more capable of assisting their pupils in a variety of difficult and problematic situations that arise in the cognitive domain. It is also apparent that the computer-based technology of virtual reality, which uses three-dimensional simulation as an instructional methodology, has many advantages over other more traditional and nontechnological instructional methodologies regarding the level of understanding attained by the learner. Thus the aim of the present study was to investigate whether instruction through the medium of a virtual reality three-dimensional simulation model effectively provides nursery school teachers with the ability to better understand children's cognitive development, perceptions and needs.

\section{METHOD}

\subsection{Sample}

The research sample consisted of 89 female nursery school teachers in their first year of teaching after obtaining a B.Ed. degree in nursery school teaching at accredited teacher training colleges. At the time of the research, during the 2001 academic year, all 89 subjects were engaged in a year-long in-service training course provided by the Division of Educational Technology in partnership with the Division of In-Service Teacher Training at the School of Education, Bar-Ilan University. During the period of their in-service training the 89 teachers agreed to participate in the present research study and 45 were randomly allocated to the experimental group and 44 to the control group. 


\subsection{Instruments}

A research questionnaire designed to examine the subjects' understanding of children's cognitive perceptions and needs was specially compiled. The first draft of the questionnaire consisted of 36 items arranged on a five-point Likert Scale and was presented to three expert university professors who specialise in nursery school teacher training for face and content validity evaluation. The items that met the validity criteria used by the expert evaluators were included in the final version of the research questionnaire.

\subsection{Hardware}

The hardware used for the virtual reality simulation included a PC Pentium III computer, a head-mounted display HMD helmet fitted with two video screens placed in the helmet eyepieces for stereoscopic vision, a stereophonic $360^{\circ} 3-\mathrm{D}$ Audio Reality kit for each ear, a virtual reality glove for changing the position of objects in the virtual world, and a tracking system for placement of the user in the virtual environment.

\subsection{Software}

The software consisted of a Super Scape $\AA$ (Version 5.5) package that managed all the virtual reality activities and interfaces. The software facilitates the building of immersive three-dimensional virtual environments that are viewed with the HMD helmet, heard when using the Audio Reality kit, and manipulated by way of the virtual reality glove.

\section{PROCEDURE}

The design of the research was quasi-experimental with a post-test only evaluation of the 89 research subjects' performance in the nursery school classroom undertaken after their period of in-service training. During their period of in-service training, each of the 45 subjects in the experimental group and the 44 subjects in the control group underwent instruction designed to increase their understanding of nursery school children's cognitive perceptions and needs according to the developmental model proposed by Piaget (1978). The content matter studied in the in-service training program emphasised the cognitive perceptions relevant to nursery school children's cognitive development, the thought processes 
characterising the level of cognitive development at the pre-operational stage, and the idiosyncratic phenomena associated with that development.

The experimental group subjects experienced 20 hours of training through the medium of a computer-based virtual reality module that simulated threedimensionally how nursery school children cognitively perceive their surroundings and their learning tasks within the nursery school environment.

Each teacher in the experimental group was transported into a threedimensional virtual environment that simulated the nursery school child's cognitive world and demonstrated how a child perceives and understands that world. Thus the experimental group subjects experienced for themselves, in a virtual environment, the cognitive problems facing nursery school children in the real world, and experienced pre-operational cognitive perceptions and needs. For example, they perceived the furniture present in the nursery school classroom from the perspective of a nursery school child and not from the perspective of an adult. The teachers' perceptions of the educational equipment such as pencils, crayons, drawing paper, and educational toys they used in the simulated virtual environment closely resembled the perceptions of nursery school children using the same equipment in their nursery school classrooms. Learning tasks, such as elementary writing and reading skills taught in the nursery school, and the construction of models using building blocks, were experienced by the teachers exactly as nursery school children experienced the same learning tasks. In this way the 45 research subjects perceived and experienced cognitive stimuli presented in the virtual world just as if they themselves were of nursery school age.

The control group participated in 20 hours of orientation workshops run by nursery school teaching instructors who conveyed to them (without the use of technology) how nursery school children perceive their cognitive world. Children's problems and cognitive difficulties were brought to the subjects' attention and they received detailed explanations regarding the children's pre-operational cognitive perceptions and needs. The subjects were instructed about nursery school children's cognitive and spatial problems and the difficulties they face when attempting the typical cognitive learning tasks undertaken at the nursery school level.

Thereafter, three Israeli Ministry of Education nursery school supervisors, who acted as evaluators of the nursery school teachers participating in the study, completed questionnaires for each of the 89 nursery school teachers in both experimental and control groups without knowing each subject's group placement.

The three supervisors were chosen as evaluators of the teachers since they were responsible for both supervision and guidance of the 89 research subjects in their respective nursery school classrooms throughout the school 
year and, in the course of their supervisory roles, met weekly with each of the 89 teachers in order to assist them with the planning and implementation of their routine teaching duties.

The task of the supervisors in the study was to evaluate the efficiency and effectiveness of the nursery school teachers' instructional abilities and their overall suitability to serve as nursery school teachers with special emphasis on their specific understanding of nursery school children's cognitive perceptions and needs.

The supervisors completed the questionnaires after observing each of the nursery school teachers in their nursery school classrooms over a period of three full teaching days after the teachers had completed the training designed to acquaint them with nursery school children's cognitive perceptions as required. In their evaluations the three supervisors specifically concentrated on the evaluation of all relevant aspects of the nursery school teachers' understanding of children's cognitive perceptions and needs in the nursery school learning situation.

After evaluating the 89 nursery school teachers in the research sample by completing a questionnaire for each research subject, the supervisors' evaluations were factor analysed in a principal components analysis and the 16 items that met the criterion of statistical significance (.30) were used in the statistical analysis of the research data. The 16 items clustered around one significant factor, which was labelled "nursery school teachers' understanding of children's cognitive perceptions". The factor had a latent root greater than unity and explained at least 10 percent of the variance. The Cronbach alpha reliability coefficient of the "nursery school teachers' understanding of children's cognitive perceptions" variable reached the 0.91 level.

\section{RESULTS}

Group means for experimental and control groups for the "nursery school teachers' understanding of children's cognitive perceptions" variable were computed from data collected from the nursery school supervisors' evaluations of the research subjects during three days of observation of how the subjects dealt with children's cognitive perceptions and needs in the course of their work. The group means were then compared in a t-test analysis calculated for independent samples. The results of the t-test are presented in Table 1. 
Table 1. T-test for independent samples for "Nursery School Teachers' Level of Understanding of Children's Cognitive Perceptions" variable

\begin{tabular}{lllllll}
\hline Group & $\mathrm{N}$ & Mean & S.D. & D.F. & t & P \\
\hline $\begin{array}{l}\text { VR } \\
\text { instruction }\end{array}$ & 45 & 41.11 & 2.49 & & & \\
$\begin{array}{l}\text { Workshop } \\
\text { instruction }\end{array}$ & 44 & 36.47 & 2.43 & 87 & 11.23 & $\mathrm{P}<0.001$ \\
\hline
\end{tabular}

Results of the t-test indicate that the nursery school teachers in the experimental group who received instruction about nursery school children's cognitive perceptions and needs through a virtual reality 3-D simulation received significantly higher evaluations of their understanding of the children's cognitive perceptions and needs than nursery school teachers belonging to the control group.

\section{DISCUSSION}

Results of the statistical analysis conducted on the data collected from the evaluations of the three nursery school supervisors of the subjects in the experimental and control groups participating in the study show that the nursery school teachers who underwent 20 hours of 3-D simulation in a virtual reality environment received superior evaluations of their understanding of children's cognitive perceptions and needs than the teachers who went through 20 hours of workshop experience on the cognitive perceptions of nursery school children. According to the supervisors' evaluations, the experimental group teachers were more sensitive to the children's cognitive needs and exhibited significantly higher levels of understanding towards them than the teachers in the control group.

The results of the study suggest that sophisticated technology used in the experiment has the advantage of immersing the adult nursery school teacher in the nursery school children's world, thereby better acquainting the teachers with that world. The result is congruent with White's (1993) research describing how learners who undertook animated simulation tasks in the physics of velocity were able to overcome common misconceptions that existed after studying the principles of velocity in a traditional nonsimulation environment, and, as a result, better understood the content matter and also seems to confirm the results of studies reported by Leary (1995) indicating that learners' achievements through the teaching medium of simulation are higher than achievements attained after undergoing other nonsimulation methods of instruction. 
The results of the study indicate that learning and instruction through the medium of a sophisticated technology platform comprising a 3-D simulation in a virtual world have the potential to prepare nursery school teachers more effectively and efficiently for their roles. Their understanding of children's cognitive perceptions is significantly superior to the skills developed when nursery school teachers learn through the medium of more traditional, nontechnological methods such as workshops.

\section{REFERENCES}

Baranauskas, M.C. and De Oliveira, O.L. (1995) Domain oriented modeling: A balance between simulation and programming. In J.D. Tinsley and T.J. van Weert (eds.) Liberating the Learner: Proceedings of the Sixth IFIP World Conference on Computers in Education, 1995. London: Chapman and Hall 119-126.

Bell, G. (1998) The personal effectiveness program initiative. Pastoral Care in Education 16 2 20-26.

Bricken, M. and Byrne, C.M. (1992) Summer Students in Virtual Reality: A Pilot Study on Educational Applications of Virtual Reality Technology. Seattle, WA: University of Washington http://www.hitl.washington.edu/projects/education/.

Calvert, M. and Henderson, J. (1994) Newly qualified teachers: Do we prepare them for their pastoral role? Pastoral Care in Education 122 7-12.

Cumming, G., Zangari, M. and Thomason, N. (1995) Designing software for cognitive change: Statplay and understanding statistics. In J.D. Tinsley and T.J. van Weert (eds.) Liberating the Learner: Proceedings of the Sixth IFIP World Conference on Computers in Education, 1995. London: Chapman and Hall 753-765.

Davies, J. (1991) Children's adjustment to nursery class: How to equalise opportunities for successful experience. School Organisation 11 255-262.

Deen, N. (1995) Schools make people grow: Notes on the supportive school. Pastoral Care in Education 133 19-25.

Glover, J.A. and Bruning, R.H. (1987) Educational Psychology: Principles and Applications. Boston: Little, Brown and Company.

Henry, D. (1992) Spatial Perception in Virtual Environments: Evaluating an ArchitecturalAapplication. Seattle, WA: University of Washington. http://www.hitl.washington.edu/projects/education/.

Katznelson, A. (1993) When it happens. Parents and Children 67 17-18 (Hebrew).

Kikas, E. (1998) The impact of teaching on students' definitions and explanations of astronomical phenomena. Learning and Instruction 8 539-454.

Leary, J.J. (1995) Computer simulated laboratory experiments and computer games: A designer's analysis. In J.D. Tinsley and T.J. van Weert (Eds.) Liberating the Learner: Proceedings of the Sixth IFIP World Conference on Computers in Education, 1995. London: Chapman and Hall 963-973.

Phillips, J.L. (1975) The Origins of Intellect: Piaget's Theory ( $2^{\text {nd }}$ ed.). San Francisco, CA: Freeman.

Piaget, J. (1978) A summary of the theory of Jean Piaget. In D.K. Gardiner (ed.) Readings in Developmental Psychology. New York: Holt 3-23. 
Regain, J.W. and Shebliske, W.L. (1992) Virtual reality: An instructional medium for visual and spatial terms. Journal of Communication 424 136-149.

Rubinstein, M. (1990) Years of growth. Tel-Aviv: Hadar Publishers (Hebrew).

Schecker, H. (1993) Learning physics by making models. Physics Education 28 102-106.

Turman, R.A. and Matton, J.S. (1994) Virtual reality: Towards improvement in simulation based training. Educational Technology 348 56-64.

Van Weert, T.J. (1995) IFIP Working Group 3.1: Towards integration of computers into education. In J.D. Tinsley and T.J. van Weert (eds.) Liberating the Learner: Proceedings of the Sixth IFIP World Conference on Computers in Education, 1995. London: Chapman and Hall 3-12.

White, B.Y. (1993) Thinker tools: Causal models conceptual change and science education. Cognition and Instruction 10 1-100.

\section{BIOGRAPHY}

Yaacov Katz is the Director of the Institute for Community Education and Research at Bar-Ilan University and serves as the Chair of the Pedagogic secretariat of the Israeli Ministry of Education. He specializes in research on attitudes of students and teachers towards different issues in the Israeli educational system, with special emphasis on attitudes toward the use of ICT in the learning and instructional processes. He has edited a number of books and written numerous scholarly papers on the above topics. He is a member of IFIP's Working Group 3.5. 